\title{
Cerebral Oximetry versus Pulse Photoplethysmography to Monitor Respiration Rate
}

\author{
I Isasi, U Irusta, E Aramendi, G Peralta, E Alonso \\ University of the Basque Country (UPV/EHU), Bilbao, Spain
}

\begin{abstract}
This study was conceived to analyze the possibility of monitoring the respiratory rate using oximetry signals obtained via Near-InfraRed Spectroscopy (NIRS) in the forehead, and to compare the results to those obtained using the photoplethysmography (PPG) signal. 29 subjects were enrolled in $30 \mathrm{~min}$ recording sessions at three controlled respiration rates: 9,12 and $18 \mathrm{~min}^{-1}$. Respiratory rate was estimated from the spectral analysis of the RR-interval time series derived from the NIRS and the PPG signals. The results show that respiratory rate was accurately determined using NIRS and PPG, although the precision was higher when PPG was used. Our results suggest that cerebral frontal lobe oximetry signals can be used for secondary analyses such as heart rate variability $(H R V)$ when NIRS signals are sampled at high rates.
\end{abstract}

\section{Introduction}

Measuring the respiratory rate may be important in several medical scenarios in which the patient's cardiorespiratory activity could be compromised, for instance in the intensive care units or in the treatment of cardiac arrest. Indeed, respiratory rate is an essential vital sign for diagnosing serious illnesses like sleep apnea or respiratory insufficiency, or even an important predictor of cardiac arrest [1]. Direct methods to measure respiratory rate, such as spirometry or capnometry, may result obtrusive and/or impractical [2]. Therefore, methods of extracting respiratory information from commonly used noninvasive physiological signals have been developed, most of them based on Respiratory Sinus Arrhytmia (RSA) [2]. This avoids the use of additional equipment and may simplify many medical scenarios.

RSA is in synchrony with respiration, and in the electrocardiogram (ECG) is seen as subtle changes in the RR intervals, which increase during expiration and decrease during inspiration. Although it is not completely clear whether RSA is an active physiological function or just reflects a passive cardiovascular response to respiratory input, physicians hypothesized that RSA is an active own function of the human body which enables the improvement of pulmonary gas exchange and circulatory efficiency [3]. RSA contributes to heart rate variability (HRV), which has been proven to be useful to estimate the respiratory rate, obtaining feedback about two vital signs with a single device [4].

Recent studies show that high-time resolution oximetry signals, primarily used to monitor patient oxygenation changes, are capable of monitoring heart rate and in turn reveal HRV [4,5]. Although the photoplethysmography (PPG) is the most widespread technique to acquire such type of signals, it is impractical in some cases such as to monitor cerebral oxygenation. In this scenario Near-Infrared Spectroscopy (NIRS) is used instead [6]. Both PPG and NIRS rest on the transmission and absorption of light as it passes through a tissue under test. Light absorption analysis allows the calculation of concentration changes in oxy $-\left(\mathrm{O}_{2} \mathrm{Hb}\right)$ and deoxy-haemoglobin $(\mathrm{HHb})$, from which tissue oxygen saturation is estimated [6]. Since high-time resolution PPG and NIRS signals provide detailed information on cardiac activity, we hypothesized that cerebral oxygenation measured using NIRS could be effectively used to monitor respiratory rate adequately. This study was conceived to analyze the possibility of monitoring the respiratory rate using NIRS signals, and to compare the results to those obtained using the PPG signal.

\section{Materials and methods}

\subsection{Materials}

Measurement system. Two independent systems were concurrently used to record the biomedical signals: (1) a NIRS sensor (PortaLite, ArtiNirs) capable of recording haemoglobin concentrations at $50 \mathrm{~Hz}$; and (2) a BioPac system equipped with an ECG (ECG 100E), PPG (OXY $100 \mathrm{E})$ and an impedance modules (NICO 100C) attached to a National Intruments data acquisition card (NI-6211) working at $250 \mathrm{~Hz}$ and $16 \mathrm{bits} / \mathrm{sample}$ per channel. Both systems were controlled using a single laptop so datafiles 
could be synchronized using time-stamps.

Measurement protocol. For each subject enrolled in the study, three 10-min sessions were consecutively recorded at controlled breathing rates: slow $\left(9 \mathrm{~min}^{-1}\right)$, normal $\left(12 \mathrm{~min}^{-1}\right)$ and fast $\left(18 \mathrm{~min}^{-1}\right)$. Subjects lay in supine position within eye contact of a visual metronome to control the breathing rate. Lead II of the ECG was recorded, thoracic impedance (TI), PPG at the left index finger, and the NIRS sensor was placed in the left hemisphere of the forehead. The ECG was used as gold standard to identify the heartbeats, and the thoracic impedance (TI) was used to monitor respiratory activity. Subjects were enrolled in the study after approval by the ethics committee of the UPV/EHU.

Final database. In total 29 subjects (20 female) were enrolled in the study. The median age, weight and height of the subjects was 24 years $(23-28.5), 67 \mathrm{~kg}$ (58-72) and $168 \mathrm{~cm}$ (163-173.5), respectively. QRS complexes were automatically detected using the Physionet version of the wqrs detector [7], and were then manually revised and audited. Breaths were identified on the TI signal as shown in Figure 1, with positive peaks indicating the end of inspiration. The time instants of the TI peaks were automatically identified and manually revised and audited.

\subsection{Methods}

First, heartbeats were detected in the PPG and the $\mathrm{O}_{2} \mathrm{Hb}$ signals. For the PPG a positive peak detector was used, and for the $\mathrm{O}_{2} \mathrm{Hb}$ a negative peak detector, as shown in Figure 1. These peak detectors were further refined using a refractory period of $0.5 \mathrm{~s}$ and a correction for false negatives if consecutive beats were not detected in a $1.3 \mathrm{~s}$ interval, the details are described in elsewhere [8].
For each of the signals an RR-interval time series was generated, $\left\{t_{n}, R R(n)\right\}$ where $n$ denotes the heartbeat number, $t_{n}$ the time position of the peak and $R R(n)=$ $t_{n}-t_{n-1}$.

The methods to estimate the respiration rate are based on the spectral analysis of the $R R(n)$ time series. However, our approach is sensitive to false positives and negatives. Therefore the $R R(n)$ time series were further refined to correct false detections. RR intervals were forced to be larger than $0.25 \mathrm{~s}$, and each RR interval was compared to the previous 3 intervals. An additional beat was added, equally separated from beats $k$ and $k-1$, if:

$$
R R(k)>1.65 \cdot \frac{1}{3} \sum_{i=k-4}^{k-1} R R(i)
$$

The $\left\{t_{n}, R R(n)\right\}$ time series was then divided into segments of 1-min duration with a $75 \%$ overlap, that is we simulated an scenario in which feedback on the respiration rate would be given every $15 \mathrm{~s}$. The power spectral density, $P_{R R}(f)$, of these segments was obtained using the Lomb-Scargle method, Figure 2 shows an example. The respiration rate was estimated as:

$$
f_{0}=\underset{f \in 6-24}{\arg \max }\left\{P_{R R}(f)\right\}\left(\min ^{-1}\right)
$$

These values were compared to the respiration rates obtained from the TI signal:

$$
\operatorname{Resp} \operatorname{Rate}\left(\min ^{-1}\right)=\frac{60}{\overline{\Delta t_{\max , i}(s)}} .
$$

where $\overline{\Delta t_{\max , i}}$ is the average time-interval between consecutive maxima of the TI signal in the segment under analysis.

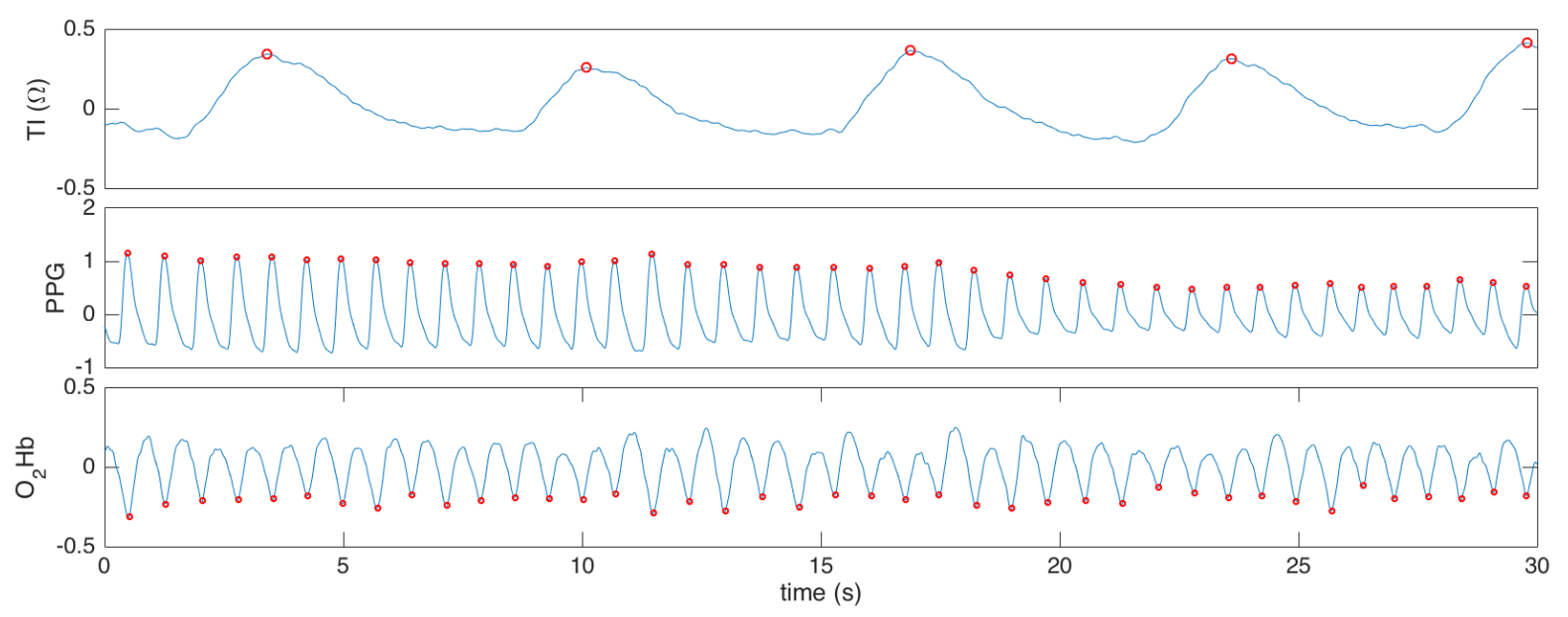

Figure 1. Example of the recorded signals showing the TI where the end of inspiration was marked to serve as gold standard for the respiration rate. RR interval time series derived from the PPG and $\mathrm{O}_{2} \mathrm{Hb}$ signals were obtained using peak detection algorithms, heartbeats were identified as positive peaks in the PPG signal and negative peaks in the $\mathrm{O}_{2} \mathrm{Hb}$ signal. 

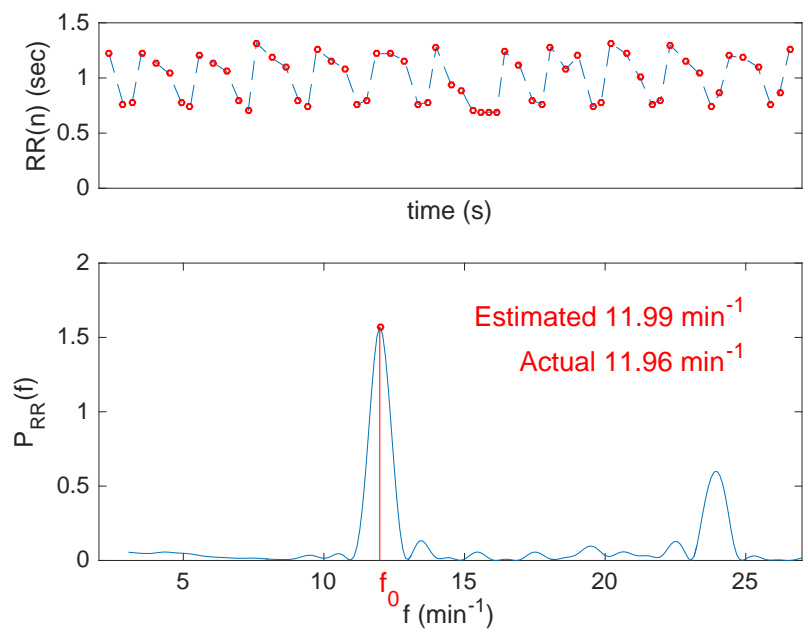

Figure 2. Estimation of the respiratory rate $\left(f_{0}\right)$ from the Lomb-Scargle spectral analysis of the RR interval time series.

\subsection{Evaluation}

The accuracy of the hearbeat detection using the $\mathrm{O}_{2} \mathrm{Hb}$ and PPG signals was evaluated in terms of sensitivity (SE) and positive predictive value (PPV). Actual heartbeat annotations were obtained from the ECG, as described in [8]. In addition, the time delay between the audited heartbeat annotations in the ECG and both the PPG and $\mathrm{O}_{2} \mathrm{Hb}$ were evaluated, because drifts in these delays may negatively influence the extraction of the RSA signal from the RR interval time series.

Respiration rates obtained from the TI were compared to those derived from the spectral analysis of the RR time series, for both the PPG and the $\mathrm{O}_{2} \mathrm{Hb}$ signals. Bland-Altman plots and their corresponding limits for the 95\% level of agreement (LOA) were used to compare the respiration rate values from the TI and the PPG or the $\mathrm{O}_{2} \mathrm{Hb}$ signals. In addition absolute errors $\left(\left|\epsilon_{r}\right|\right)$, absolute relative errors $\left(\left|\epsilon_{r}\right|\right)$, and the limiting values for $90 \%$ of the absolute errors $\left(P_{90}\right)$ were determined.

\section{Results}

A total of $30 \mathrm{~min}$ were recorded per subject, and in total there were 57252 heartbeats. Hearbeats were detected with a SE of $99.88 \%$ and a PPV of $99.97 \%$ on the PPG, and a SE of $99.68 \%$ and a PPV of $99.82 \%$ on the $\mathrm{O}_{2} \mathrm{Hb}$ signal. Consequently, the number of false positive/negative detections were 19/68 for the PPG and $102 / 181$ for the $\mathrm{O}_{2} \mathrm{Hb}$ signal. The mean delay from the QRS onset and the peaks detected in the PPG and $\mathrm{O}_{2} \mathrm{Hb}$ signals (see Figure 1) were $459 \pm 36 \mathrm{~ms}$ and $489 \pm 46 \mathrm{~ms}$, respectively.

The Bland-Altman plots for the respiration rates

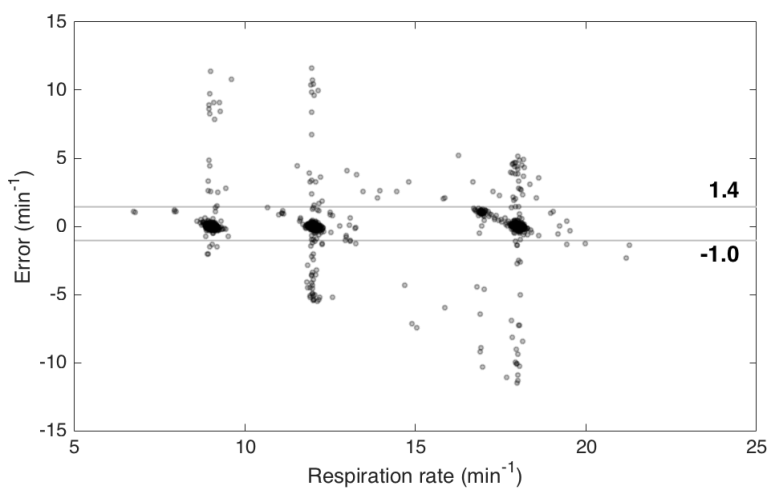

Figure 3. Bland-Altman plot comparing the respiration rates obtained from the PPG signal, and from the reference respiration marks derived from the TI signal. The horizontal lines show the $95 \%$ LOA. Values are concentrated around the three controlled respiration rates $\left(9,12,18 \mathrm{~min}^{-1}\right)$.

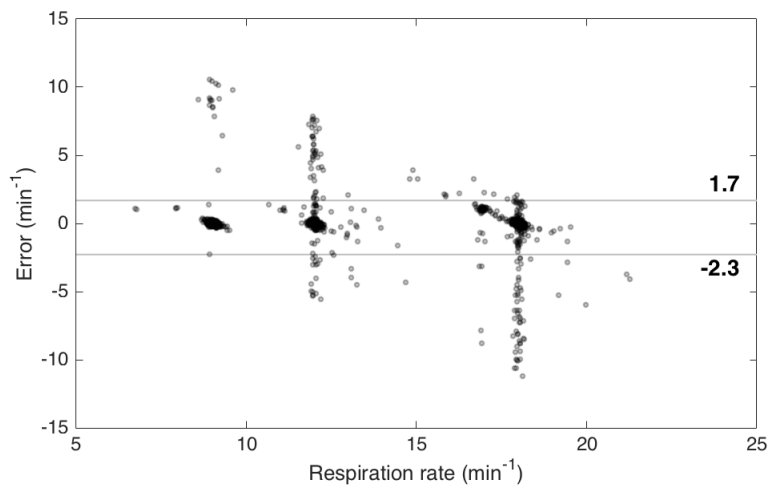

Figure 4. Bland-Altman plot comparing the respiration rates obtained from the $\mathrm{O}_{2} \mathrm{Hb}$ signal, and from the reference respiration marks derived from the TI signal.

obtained from the PPG and $\mathrm{O}_{2} \mathrm{Hb}$ signals are shown in Figures 3 and 4, respectively. Respiration rate feedback based on the PPG signal was more accurate with $95 \%$ LOAs of $\left(-1.0-1.4 \mathrm{~min}^{-1}\right)$, compared to the $\left(-2.3-1.7 \mathrm{~min}^{-1}\right)$ obtained for the $\mathrm{O}_{2} \mathrm{Hb}$ signal. The mean absolute errors and relative errors for the PPG signal were $0.34( \pm 1.23)$ and $2.62( \pm 9.64) \%$, respectively; and 0.41 $( \pm 1.34)$ and $3.11( \pm 10.71) \%$ for the $\mathrm{O}_{2} \mathrm{Hb}$ signal. The detailed values of the errors for all respiration rates are shown in Table 1. In general errors are small, under $3.5 \%$.

\section{Discussion}

This study shows that respiration rate calculations obtained from the HRV analysis of RSA are comparable when using oximetry signals recorded via the standard PPG on the index finger and a NIRS system to measure cerebral oxygenation. The accuracy on respiration rate 


\begin{tabular}{lcrcc}
\hline Signal & $|\epsilon|,\left(\mathrm{min}^{-1}\right)$ & \multicolumn{1}{c}{$P_{90}$} & $\left|\epsilon_{r}\right|(\%)$ & $P_{90}$ \\
\hline $\mathbf{P P G}$ & & & & \\
$9 \mathrm{~min}^{-1}$ & $.20(.95)$ & .19 & $2.3(10.4)$ & 2.1 \\
$12 \mathrm{~min}^{-1}$ & $.35(1.20)$ & .31 & $2.9(10.0)$ & 2.5 \\
$18 \mathrm{~min}^{-1}$ & $.48(1.47)$ & 1.00 & $2.7(8.4)$ & 5.9 \\
$\mathbf{O}_{\mathbf{2}} \mathbf{H b}$ & & & & \\
$9 \mathrm{~min}^{-1}$ & $.23(1.16)$ & .18 & $2.6(12.9)$ & 2.0 \\
$12 \mathrm{~min}^{-1}$ & $.42(1.21)$ & .60 & $3.5(10.1)$ & 5.0 \\
$18 \mathrm{~min}^{-1}$ & $.57(1.58)$ & 1.19 & $3.2(8.83)$ & 6.9 \\
\hline
\end{tabular}

Table 1. Precision of the respiration rate feedback for the different respiration rates, errors as mean (standard deviation).

feedback was comparable for the PPG and the $\mathrm{O}_{2} \mathrm{Hb}$ signals, although feedback using the PPG was more precise for all breathing conditions. Several factors explain this higher accuracy. First, heartbeat detection was more accurate using the PPG, with 3 times less false detections than in the $\mathrm{O}_{2} \mathrm{Hb}$ signal. Heartbeat detection is more challenging in NIRS signals due to artefacts and larger oscillations in the beat-to-beat amplitude. Second, the deviation in the delay from the ECG fiducial point to the detected peak was larger in the $\mathrm{O}_{2} \mathrm{Hb}$, indicating that the time instants of the heartbeats were detected with a lower precision. Again this was caused by noise in the NIRS signal appearing as multiple negative peaks but also to the lower sampling rate used for the NIRS signal, which is an intrinsic limitation of the hardware used for the study. These two factors combined resulted in a lower quality $\mathrm{RR}$ interval time series from the $\mathrm{O}_{2} \mathrm{Hb}$ that explains the slightly lower accuracy when determining the respiratory rate using the HRV analysis of RSA.

Our results also show that higher sampling rates for cerebral oxygenation signals acquired using NIRS technology may enable several secondary analyses. Indeed, our experimental protocol was conceived to determine respiratory rate, which was estimated within reasonable accuracy. However, many additional analyses could be carried out, starting with standard HRV analyses. Therefore, it would be interesting to increase the sampling rates of current clinical NIRS devices, so that haemoglobin concentration signals at sampling rates above $50 \mathrm{~Hz}$ are at least internally stored.

Finally, there are some limitations to the methods and the protocol used in this study. First, data was gathered on favourable conditions on young healthy subjects, who generally present stronger RSA. In addition, only data on controlled respiration rates (visual metronome) was analyzed. More challenging patient and respiration conditions may compromise the accuracy of our methods.

\section{Acknowledgements}

This work received financial support from Spanish Ministerio de Economía y Competitividad, project TEC2012-31928, and jointly with the Fondo Europeo de Desarrollo Regional (FEDER), project TEC2015-64678-R; from UPV/EHU via unit UFI11/16 and project AE15/01.

\section{References}

[1] Cretikos MA, Bellomo R, Hillman K, Chen J, Finfer S, Flabouris A. Respiratory rate: the neglected vital sign. Med J Aust Jun 2008;188(11):657-659.

[2] Karlen W, Brouse CJ, Cooke E, Ansermino JM, Dumont GA. Respiratory rate estimation using respiratory sinus arrhythmia from photoplethysmography. Conf Proc IEEE Eng Med Biol Soc 2011;2011:1201-1204.

[3] Hayano J, Yasuma F, Okada A, Mukai S, Fujinami T. Respiratory sinus arrhythmia. a phenomenon improving pulmonary gas exchange and circulatory efficiency. Circulation Aug 1996;94(4):842-847.

[4] Garde A, Karlen W, Ansermino JM, Dumont GA. Estimating respiratory and heart rates from the correntropy spectral density of the photoplethysmogram. PLoS One 2014; 9(1):e86427.

[5] Lázaro J, Gil E, Bailón R, Mincholé A, Laguna P. Deriving respiration from photoplethysmographic pulse width. Med Biol Eng Comput Feb 2013;51(1-2):233-242.

[6] Ghosh A, Elwell C, Smith M. Review article: cerebral near-infrared spectroscopy in adults: a work in progress. Anesth Analg Dec 2012;115(6):1373-1383.

[7] Zong W, Moody GB, Jiang D. A robust open-source algorithm to detect onset and duration of QRS complexes. In Computers in Cardiology, 2003. 2003; 737-740.

[8] Isasi I, Irusta U, Aramendi. E, Peralta G, Alonso E. Monitoring the heart rate in cerebral oximetry signals. In Computing in Cardiology, 2016. 2016; .

Address for correspondence:

Name: Iraia Isasi Liñero

Full postal address: Alda Urquijo s/n, 48013,Bilbao,Spain

E-mail address: iraiaehu@gmail.com 